\title{
Décisions médicales justifiées sous le couvert de la libre appréciation: variations non nécessaires
}

\author{
Niranjan Kissoon, MD
}

Le pouvoir de la libre appréciation des médecins comme fondement des prises de décision est considéré comme un pilier sacro-saint de la notion de la médecine en tant qu'art. Toutefois, ce pouvoir de la libre appréciation s'acquiert au fil du temps par la formation et les expériences passées; il est donc susceptible d'être teinté de préjugés inhérents. Ainsi, le pouvoir de la libre appréciation poussé à l'excès conduit à des variations non nécessaires de demandes d'examens de diagnostic et de traitements, et se dresse en abomination contre les principes de qualité et de sûreté en médecine. Les différences d'approche diagnostique à l'égard d'une affection particulière ne manquent pas entre les disciplines médicales et au sein de mêmes disciplines; il n'y a rien de nouveau làdedans. Alors, pourquoi publier le rapport de Guttmann et de ses collaborateurs dans le présent numéro de la revue? ${ }^{1}$

Guttmann et ses collaborateurs ont analysé les demandes de radiographies chez les enfants souffrant d'asthme, de croup, ou de bronchiolite avérés, qui ont été examinés dans l'un ou l'autre des 164 services d'urgence (SU), en Ontario, et qui ont par la suite obtenu leur congé. Les auteurs ont constaté que les enfants examinés aux SU dotés de personnel spécialisé en soins pédiatriques de première ligne étaient moins susceptibles d'être soumis à des radiographies que ceux qui étaient vus dans des hôpitaux dotés de pédiatres consultants, constatation qui s'est montrée insensible à l'accès aux protocoles cliniques. Peut-être que les protocoles en question ne précisaient dans quels cas il fallait demander une radiographie pulmonaire. Les points forts de cette étude sont la taille: $164 \mathrm{SU}$, et un taux de réponse de $100 \%$. Autre point tout aussi important: le fait que les auteurs se sont penchés sur une population peu malade (renvoyée du SU), souffrant d'affections fréquentes chez les enfants. Aussi pourrait-on s'attendre, de prime abord, à ce que l'évaluation clinique dans ce groupe de patients donne lieu à moins d'écarts.

Or, les résultats indiquent un écart d'au moins du triple entre les centres pédiatriques et les centres non pédiatriques; la constatation est troublante et peut être un signe révélateur de problèmes plus profonds, liés à la pratique. Supposons, un instant, que l'hypothèse de l'auteur sur les demandes abusives soit juste. Il ne s'agit pas d'un problème purement théorique; bien que l'exposition au rayonnement émis par les radiographies pulmonaires soit minime, la fréquence accrue de l'emploi des antibiotiques, ${ }^{2}$ l'augmentation des coûts, et le prolongement des délais d'attente, eux, ne sont pas banals dans le contexte de SU achalandés. De plus, les demandes abusives de radiographies pulmonaires représente la pointe de l'iceberg et peut être le reflet d'une pratique selon laquelle ces mêmes praticiens demanderaient inutilement des examens plus chers et plus nocifs ( $\mathrm{p}$. ex., les tomodensitométries de la tête ou de l'abdomen). Par contre, d'après les données exposées, nous ne pouvons pas non plus présenter les demandes abusives comme un problème étant donné que nous ne disposons pas des données sur les variations entre spécialités ni de critère pour juger des pratiques exemplaires en matière de

\footnotetext{
See the related article in this issue on page 8.
}

From BC Children's Hospital and Sunny Hill Health Centre for Children and Department of Pediatrics and Emergency Surgery, University of British Columbia; Child and Family Research Institute, Vancouver BC; and World Federation of Paediatric Intensive and Critical Care Societies, Geneva, Switzerland.

Demandes: Dr. Niranjan "Tex" Kissoon, BC Children's Hospital and Sunny Hill Health Centre for Children and Department of Pediatrics and Emergency Surgery, University of British Columbia, B24C - 4480 Oak Street, Vancouver, BC V6H 3V4.

L'article a été évalué par les pairs. 
prescription des radiographies pulmonaires. L'un des points faibles possibles de l'étude est la collecte de données sur les diagnostics inscrits au moment du congé du SU; ceux-ci sont choisis arbitrairement par les médecins, sans critère particulier. Il est donc possible que les données de l'étude soient faussées par l'inscription d'autres diagnostics: syndrome viral, pneumonie, fièvre, bronchite, infection virale des voies respiratoires supérieures. Alors, il serait même possible d'imaginer un scénario dans lequel un pédiatre demanderait une radiographie pulmonaire pour de l'asthme et qui, en voyant des stries sur l'image, inscrirait le diagnostic de pneumonie au moment du congé.

L'application de protocoles aux fins d'une utilisation rationnelle des examens peut diminuer les écarts et les demandes abusives. ${ }^{3}$ L'absence de diminution du nombre de radiographies dans les centres dotés de protocoles peut s'expliquer par la non-application des protocoles ou par des critères relatifs aux pratiques exemplaires non suffisamment explicites au regard des demandes de radiographies. Quoi qu'il en soit, des écarts importants d'opinions et de pratiques de la part de cliniciens, comme en font état le présent rapport et d'autres documents, sont inacceptables; pourtant, ils sont largement tolérés.,5 Nous ne pouvons rester en terrain neutre et accepter des écarts importants de pratique pour des affections courantes. Il faudrait considérer les variations comme une abomination contre les bonnes pratiques mais, en même temps, éviter de proposer des solutions qui découlent de réactions émotives parce qu'elles seraient, au mieux, inutiles, et, au pire, préjudiciables. Aussi faut-il d'abord comprendre les raisons sous-jacentes à ces différences de pratique avant de proposer des solutions.

On peut probablement imputer les variations aux préférences des médecins en matière de pratique, aux opinions véhiculées dans les lignes directrices, aux lacunes en ce qui concerne l'application des connaissances, à la formation et à la culture locale. ${ }^{4-7}$ L'application des connaissances-depuis les lignes directrices jusqu'à la pratique-a toujours été un domaine dans lequel la profession médicale a connu des succès mitigés. Si l'application des connaissances est le problème principal, alors il nous appartient de nous concerter pour régler ce genre de problèmes à grande échelle. Pour ce faire, il faudrait, par exemple, formuler des lignes directrices appropriées, fondées sur des données probantes ou sur l'avis d'experts en cas d'insuffisance de données probantes, et lier ces lignes directrices aux protocoles, aux pratiques courantes de fonctionnement, et aux ensembles de modèles d'ordonnances. ${ }^{8}$ Il faudrait aussi veiller à ce qu'il soit possible de ne pas suivre ces lignes directrices plutôt que de les choisir et que les écarts de lignes directrices soient évalués dans un cadre d'assurance de la qualité. Il faudrait, en outre, comprendre les raisons pour lesquelles les médecins choisissent de ne pas appliquer les lignes directrices et s'attaquer aux questions de perception ou de barrière culturelle. ${ }^{9}$ Si les variations sont attribuables à la formation, alors il faudra se pencher sur la formation. Il n'existe pas de panacée, et une approche à facettes visant à atténuer les écarts sera sans doute nécessaire.

L'affirmation de Guttmann et de ses collaborateurs selon laquelle les demandes abusives posent un problème, et l'hypothèse selon laquelle le problème va bien au-delà des radiographies pulmonaires sautent aux yeux. Les demandes abusives d'examens comportent peu d'avantages et beaucoup d'inconvénients. D'ailleurs, le fait d'avoir évité la question pour des affections courantes et relativement simples me laisse perplexe. Nous sommes tous coupables d'avoir fermé les yeux sur les demandes abusives d'examens et de traitements. Le clinicien se cache sous le couvert de l'« autonomie » pour éviter l'application de protocoles et de lignes directrices, malgré leurs avantages reconnus pour les familles des patients et pour l'efficacité des services. Certains médecins demandent plus d'examens que nécessaire en raison de leur crainte non fondée selon laquelle la réalisation de plusieurs examens se traduira par une diminution du risque de poursuite ou d'erreur, mais ni l'une ni l'autre de ces appréhensions ne reposent sur des assises. De plus, une grande partie du taux de variation en ce qui concerne les demandes d'examens n'est que le reflet objectif du degré variable d'aversion que chaque médecin a à l'égard du risque. Chacun, chacune d'entre nous connaît un ou une collègue qui demande plus d'examens, qui est moins efficace ou qui a une plus grande aversion pour le risque que soi. Mais le principe d'une pratique médicale sûre ne devrait-il pas primer toute autre considération? Si l'aspect juridique est si important en médecine, est-ce que la collectivité juridique pourrait ou devrait donner son appui aux lignes directrices? Par ailleurs, selon d'autres données, les médecins demanderaient plus d'examens parce qu'ils passent moins de temps avec les patients ou 
qu'ils essaient d'être à la hauteur des attentes des patients. Une sérieuse réflexion s'impose sur ces questions.

Les lignes directrices en matière de pratique ne devraient pas primer le bon jugement, et des différences d'approche en ce qui concerne les soins sont acceptables. Nous n'avons guère besoin d'autres données pour confirmer l'existence de variations. Il faut aller au-delà de la recherche qui se borne au dépouillement de bases de données ou à la collecte de données pour confirmer l'existence de variations. ${ }^{1,10}$ Les auteurs des études doivent également examiner si ces variations ont des conséquences défavorables et proposer des solutions. Si les variations se soldent par des soins de moindre qualité et des résultats cliniques désavantageux, alors nous devrions tous monter au créneau et nous engager à agir.

Reconnaître le problème est facile, mais il en va tout autrement des changements de comportement à adopter pour assurer une prestation optimale des soins, tant sur le plan de la qualité que sur celui de la valeur. Tout d'abord, les cliniciens ont besoin d'élaborer des lignes directrices fondées sur de solides données probantes et sur l'expérience et de s'assurer qu'elles seront mises en œuvre et largement appliquées. Ensuite, les barrières culturelles à une mise en œuvre réussie sont énormes, et il nous faudra, entre autres, faire appel à des anthropologues et à des guides d'opinion. De leur côté, les chercheurs, y compris les économistes de la santé, ont besoin de déterminer si le fait de s'écarter des lignes directrices se traduit par une diminution de la qualité des soins et par une augmentation des coûts. Certes, il s'agit là de tâches difficiles, mais jouer à l'autruche ne les fera pas disparaître comme par enchantement, et personne d'autre ne voudra porter sur ses épaules le poids de la recherche de solutions à ces problèmes. Si nous ne nous y attaquons pas, qui le fera?
Intérêts concurrentiels: aucun intérêt déclaré.

Keywords: children, emergency medicine, guidelines, quality, radiology

\section{RÉFÉRENCES}

1. Guttmann A, Weinstein M, Austin P, et al. Variability in the emergency department use of discretionary radiographs in children with common respiratory conditions: the mixed effect of access to pediatrician care. CFEM 2012.

2. Schuh S, Lalani A, Allen U, et al. Evaluation of the utility of radiography in acute bronchiolitis. 7 Pediatr 2007;150:42933, doi:10.1016/i.jpeds.2007.01.005.

3. Gentile NT, Ufberg J, Barnum M, et al. Guidelines reduce s-ray and blood gas utilization in acute asthma. Am 7 Emerg Med 2003;21:451-3, doi:10.1016/S0735-6757(03)00165-7.

4. Battle CE, Hutchings H, Evans PA. Expert opinion of the risk factors for morbidity and mortality in blunt chest wall trauma: results of a national postal questionnaire survey of emergency departments in the United Kingdom. Injury 2012 Jan 6. [Epub ahead of print]

5. Chamberlain MC, Reid SR, Madhok M. Utilization of emergency ultrasound in pediatric emergency departments. Pediatr Emerg Care 2011;27:628-32, doi:10.1097/PEC. 0b013e3182259908.

6. Lougheed MD, Garvey N, Chapman KR, et al. Variations and gaps in management of acute asthma in Ontario emergency departments. Chest 2009;135:724-36, doi:10. 1378/chest.08-0371.

7. Knapp JF, Hall M, Sharma V. Benchmarks for the emergency department care of children with asthma, bronchiolitis and croup. Pediatr Emerg Care 2010;26:364-9, doi:10.1097/PEC.0b013e3181db2262.

8. Grol R, Grimshaw J. From best evidence to best practice: effective implementation of change in patients' care. Lancet 2003;362:1225-30, doi:10.1016/S0140-6736(03)14546-1.

9. Scott DS, Grimshaw J, Klassen TP, et al. Understanding implementation processes of clinical pathways and clinical practice guidelines in pediatric contexts: a study protocol. Implement Sci 2011;6:133, doi:10.1186/1748-5908-6-133.

10. Richer LP, Laycock K, Millar K, et al. Treatment of children with migraine in emergency departments: national practice variation study. Pediatrics 2010;126:e150-5, doi:10. 1542/peds.2009-2337. 